\title{
A Patient with Chronic Hepatitis $C$ and a History of Abuse of Analeptic Drugs, Who Showed Hallucination and Delusion with Interferon Administration
}

\author{
HARUO MURAOKA, TOSHIAKI SANEFUJI, RYUJI KEIDA, RIKO TSUJI, \\ HIROHIKO ABE, YASUYO UCHIMURA* , MICHIO SATA* , KYUICHI TANIKAWA*, \\ IWAO NOSE ${ }^{* *}$, NAOTAKA UCHIMURA** AND YOICHI NAKAZAWA** \\ Department of Internal Medicine, Kumamoto Central Hospital, Ozu 869-12 and \\ Departments of Medicine* and Neuropsychiatry ${ }^{* *}$, Kurume University School of \\ Medicine, Kurume 830, Japan
}

Received for publication September 6, 1995

\begin{abstract}
Summary: We experienced a case of chronic hepatitis type C accompanied with hallucination and delusion induced by interferon (IFN) therapy positive. The case was a 47-year-old male, whose laboratory data showed positive for antiHepatitis C Virus (HCV) and elevated transaminase level. He was treated with 6 MU/day of natural-type IFN- $\alpha$ (HLBI). Sleeplessness and delusions of persecution developed about 2 months after the start of IFN therapy. The interview of the psychiatrist disclosed that the patient had a history of addiction to drugs, and these psychiatric symptoms were diagnosed as being of "the flashback phenomenon." These side effects were improved after the administration of psychotropics and it was suggested that we had to take care of the development of flashback phenomenon during the treatment of IFN in cases of chronic hepatitis with a history of addiction to drugs.
\end{abstract}

Key words: flash back - interferon - hallucination - analeptic drugs delusion

\section{Introduction}

It is well known that mental symptoms and signs, such as depression and abnormal electroencephalographic (EEG) findings (Renault et al. 1987; Merimsky and Chiaitchick, 1992), develop with interferon (IFN) administration. However, to our knowledge, there have been no reports on recrudescence (flashback) (Utena, 1982) of symptoms in patients with analeptic drug intoxication. We encountered a patient with chronic hepatitis C, who had abused analeptic drugs in the past, and who developed hallucinations and was delusional during IFN administration, suggestive of flashbacks. We considered the flashbacks to be a side effect of IFN therapy, to which attention should be paid in the future along with the widespread use of IFN therapy.

Reprint requests to: Haruo Muraoka, Department of Internal Medicine, Kumamoto Central Hospital, 955 Ozu-machi, Kikuchi-gun, Kumamoto 869-12, Japan. Tel: 81-96-293-0555 Fax: 81-96-293-2562 


\section{Case Report}

Patient: A 47-year-old man.

Chief complaints: Sleeplessness and anxiety.

Family history: His brother had a brain tumor. No psychiatric hereditary factors were identified.

Past history: Appendectomy at 21 years of age. No past history of psychiatric diseases.

Premorbid personality: Sociable and cheerful.

Present condition: Hepatic dysfunction was discovered by screening on July 21 , 1993, and he visited our outpatient clinic on August 25. He was found to be positive for hepatitis $\mathrm{C}$ virus (HCV) antibody. On August 30, he was admitted to our hospital, and underwent liver biopsy. The histological findings of the liver showed chronic active hepatitis. For IFN therapy, he was re-admitted to our hospital on September 9.

Present illness on admission: His consciousness was clear, and there were no particular abnormalities in regards to mental condition. He was $166 \mathrm{~cm}$ in height and weighed $50 \mathrm{~kg}$. There was no anemia or jaundice. The liver was palpable at 1 fingerbreadth above the right midclavicular line and at 3 fingerbreadths in the epigastrium.

Results of testing on admission (Table 1): Serum GOT and GPT were slightly elevated, and biliary tract enzymes and the colloid reaction were slightly elevated. HBs antigen and HBs antibody were both negative and $\mathrm{HCV}$ antibody was positive, on examination for hepatitis virus markers. The HCV-RNA level was $10^{3.5} \mathrm{copy} / \mathrm{ml}$ by the RT-polymerase chain reaction $(\mathrm{PCR})$ method.
TABLE 1.

Laboratory data on admission

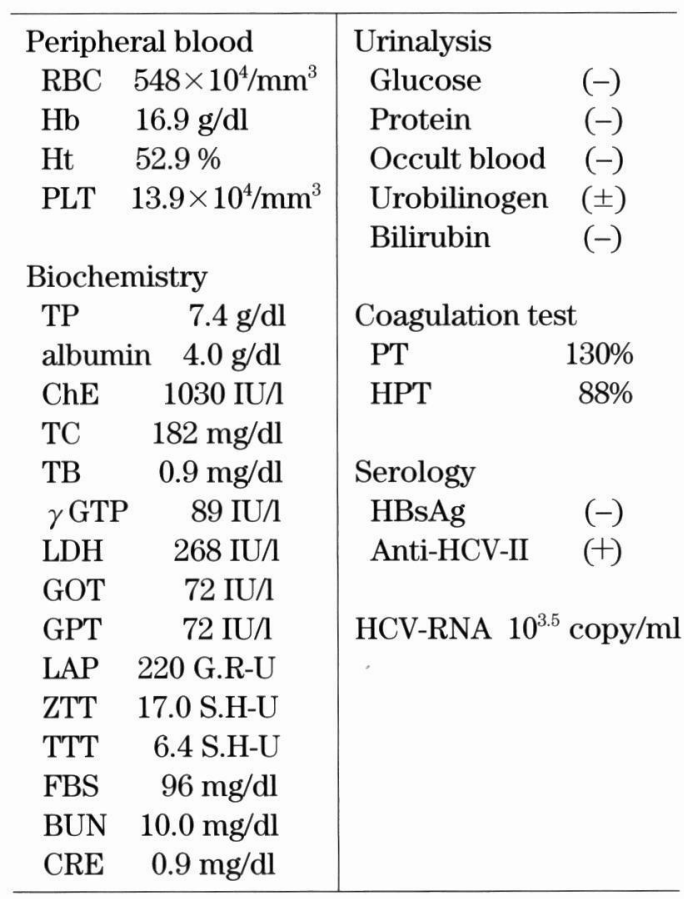

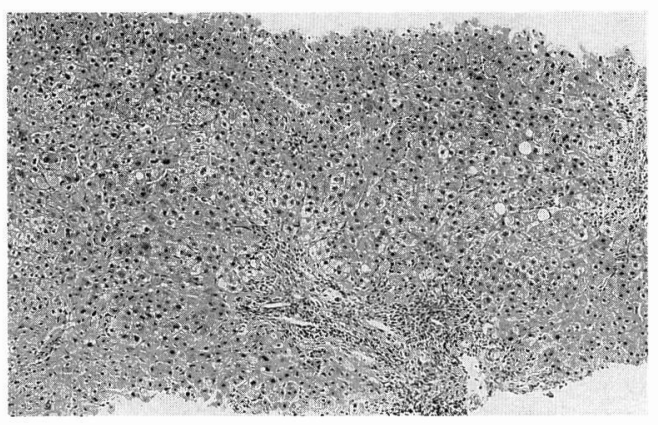

Fig. 1. Histological finding of the case showing chronic active hepatitis. (HE, $\times 50)$

Liver biopsy (Fig. 1): There were findings of chronic active hepatitis, including round cell infiltration of the portal area, piecemeal necrosis and focal necrosis. Clinical course (Fig. 2): Administration of IFN was started, according to the following schedule, on September 10; 


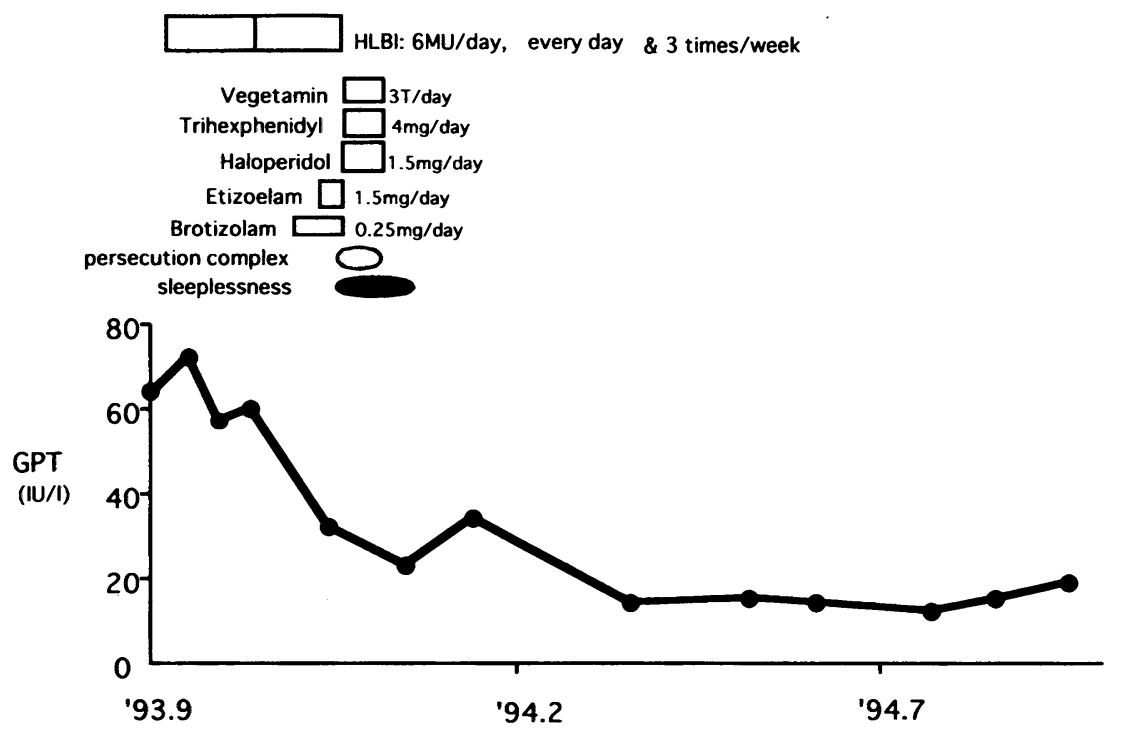

Fig. 2. Clinical course.

natural-type IFN- $\alpha$ (HLBI) was intramuscularly injected at a daily dose of $6 \mathrm{MU}$ for 4 successive weeks, and was subsequently scheduled to be administered three times a week for 20 weeks (total dose was $528 \mathrm{MU}$ ). The patient felt chilled, and had pyrexia and a sense of weariness beginning on the first day of administration, but these symptoms improved with an antipyretic (suppository). Since sleeplessness appeared on November 9, when the total dose of HLBI was $252 \mathrm{MU}$, administration of a sleep inducer ( $0.25 \mathrm{mg}$ of brotizolam) was initiated. However, the sleeplessness showed no improvement, and in late November when the total dose was 270 MU, the patient had a persecution complex and a visual hallucination, saying "I'm being chased by the police." Since the possibility of mental symptoms attributable to IFN was considered,
HLBI administration was discontinued on November 24, when the total dose was $282 \mathrm{MU}$, and he was referred to the Department of Psychiatry of our hospital. The results of the psychiatric examination revealed that the patient had abused analeptic drugs for half a year 20 years previously, according to a statement by the patient himself. The possibility of flashback was considered, and administration of psychotropics (1.5 mg of haloperidol and $3 \mathrm{~T}$ of Vegetamin-A) was started on November 30. The symptoms gradually improved; persecution complex had subsided by December 7, and the sleeplessness had resolved by the end of December. Subsequently, the patient was examined in an outpatient clinic, without readministration of HLBI. The GPT level was normalized to $32 \mathrm{IU} / \mathrm{L}$ on November 6, and the GPT level thereafter showed changes within the normal 
range and no mental symptoms have since appeared.

\section{Discussion}

IFN is known to exhibit toxic effects on the central nervous system when administered at massive doses for the treatment of patients with malignant tumors (Merimsky and Chiaitchick, 1992). Lethargy, insanity, paresthesia, dyskinesia, disorientation, and hallucination are among the central nervous system symptoms encountered. EEG revealed slowing of the $\alpha$ wave. These symptoms have been considered to occur due to hypermetabolism of brain tissue arachidonic acid by IFN and neurotransmitter hyperactivity due to IFN, but the precise mechanism remains unknown. Side effects of IFN are most likely to occur with massive administration and in the elderly. The frequency of side effects is thought to be high when the primary lesion is renal cell carcinoma, in malignant tumor cases (Merimsky and Chiaitchick, 1992). Recently, however, a few reports have shown, along with the increasing application of IFN therapy for chronic hepatitis $\mathrm{B}$ and $\mathrm{C}$, that psychonervous symptoms appear with IFN administration even at relatively small doses (Renault et al. 1987; Uchimura et al. 1994; Yokoyama et al. 1994).

The details of psychonervous symptoms in patients with chronic hepatitis treated with IFN have been reported by Renault et al. (1987) in a series of 58 patients with chronic hepatitis $\mathrm{B}$, and by Yokoyama et al. (1994), in a series of 43 patients with chronic hepatitis C. With regard to the incidence of mental symptoms, Renault et al. (1987) reported an incidence of $17 \%, 10$ of 58 patients with chronic hepatitis B. Yokoyama et al. (1994) noted differences in the incidence among hospitals, i.e., 0-36\%, but described mental symptoms as having appeared in 4 (25\%) of their 16 patients. From these reports, mental symptoms are considered to be relatively common. According to Yokoyama et al. (1994), the incidence of depression is the highest among mental symptoms, but hallucination and delusion are also observed, although at low incidences. The hallucinations and delusional behavior observed in our patient might have been the direct effects of IFN. Renault et al. (1987) reported that the total dose of IFN in patients in whom mental symptoms appeared correlated with the dose. In contrast, Yokoyama et al. (1994) described mental symptoms as possibly appearing even at a small dose, as with the incidences of mental symptoms in general. Mental symptoms appeared in the present patient at the relatively small dose of 270 MU. This supports the observations of Yokoyama et al. (1994). With regard to the time of appearance of mental symptoms, Renault et al. (1987) reported 1-3 months after administration, while Yokoyama et al. (1994) reported the appearance to be within one month in $67 \%$ of cases. Thus, mental symptoms are considered to appear relatively early, i.e., within one month, after IFN administration in many patients. The present patient showed mental symptoms about 2 months after starting administration. The appearance of mental symptoms. should always be kept in mind during IFN administration. 
The mental symptoms in the present patient might also have been flashbacks (Utena, 1982) as well as a direct effect of the IFN, because the patient had a past history of abuse of analeptic drugs.

Flashback is believed to be a phenomenon occurring in patients who have a history of chronic intoxication with analeptic drugs, in which toxic symptoms such as acute hallucination and delusion are exacerbated by the use of a small amount of an analeptic drug or psychological stress (Utena, 1982). It is thought to be very similar to the symptoms of schizophrenia. Various factors are believed to induce flashbacks: alcohol consumption, sleeplessness, an irregular daily cycle showing a day-night reversal, extreme physical fatigue, the persistence of stressful situations such as tension and irritation for several weeks. The present patient had a past history of chronic intoxication with analeptic drugs 20 years prior to IFN treatment, but had not previously experienced a flashback. In the present study, the patient underwent frequent intramuscular administration of IFN, and he had anxiety regarding the chronic underlying disease in addition to physical fatigue manifesting as pyrexia and a sense of general weariness, which were attributed to IFN, in addition to the stress of sleeplessness. It is probable that the flashbacks appeared as a result. On the other hand, Sato et al. (1982) reported that flashback occurs in the presence of hypersensitivity of the brain which has resulted from the abuse of analeptic drugs. It is therefore speculated that mental symptoms are more likely to be induced by IFN in patients who have such a biological background. In any event, abuse of analeptic drugs is considered to be a major contributor to the appearance of hallucination and delusion following IFN administration.

The incidence of depression as the mental symptom occurring after IFN administration is generally the highest, but in patients with a past history of analeptic drug abuse, particular attention should be paid to the appearance of hallucination and delusion, the hallmarks of the flashback phenomenon.

\section{References}

Merimsky O, and Chiaitchick S. Neurotoxicity of interferon- $\alpha$. Anti-Cancer Drugs 1992; 3: 567-570.

Renault PF, Hoofnagle JH, Park Y, Mullen KD, Peters M et al. Psychiatric complication of long-term interferon therapy. Arch Intern Med 1987; 147:1577-1580.

Sato M, Akiyama K, Nakashima T, Otsuki S, Harada $\mathrm{T}$ et al. Reverse tolerance phenomenon in the clinical course of chronic Methamphetamine psychosis and prophylatic effect of anti-psychotics on relapse by re-use of methamphetamine. Clinical Psychiatry 1982; 24:1333-1340. (in Japanese)

Uchimura N, Nakashima J, and Motohka H. Psychiatric symptoms following interferon administration. Japanese Journal of Clinical Psychiatry 1994; 23: 1105-1111. (in Japanese)

Utena $\mathrm{H}$. A phenomenon of recrudescence of intoxication with analeptic drugs-- Flashback. In: Seishinka MOOK, ed. Kato N, Kanehara Shuppan, Tokyo, No 3, pp 70-78, 1982. (in Japanese)

Yokoyama A, Takagi S, Nakamura H, Miyairi M, Oda $\mathrm{Y}$ et al. Mental symptoms during interferon treatment for chronic hepatitis. Japan Medical Journal 1994;3638:43-46. (in Japanese) 